\title{
Approximation of Upper Lyapunov Exponents of Bilinear Stochastic Differential Systems
}

\author{
Denis Talay
}

SIAM Journal on Numerical Analysis, Vol. 28, No. 4. (Aug., 1991), pp. 1141-1164.

Stable URL:

http://links.jstor.org/sici?sici=0036-1429\%28199108\%2928\%3A4\%3C1141\%3AAOULEO\%3E2.0.CO\%3B2-D

SIAM Journal on Numerical Analysis is currently published by Society for Industrial and Applied Mathematics.

Your use of the JSTOR archive indicates your acceptance of JSTOR's Terms and Conditions of Use, available at http://www.jstor.org/about/terms.html. JSTOR's Terms and Conditions of Use provides, in part, that unless you have obtained prior permission, you may not download an entire issue of a journal or multiple copies of articles, and you may use content in the JSTOR archive only for your personal, non-commercial use.

Please contact the publisher regarding any further use of this work. Publisher contact information may be obtained at http://www.jstor.org/journals/siam.html.

Each copy of any part of a JSTOR transmission must contain the same copyright notice that appears on the screen or printed page of such transmission.

The JSTOR Archive is a trusted digital repository providing for long-term preservation and access to leading academic journals and scholarly literature from around the world. The Archive is supported by libraries, scholarly societies, publishers, and foundations. It is an initiative of JSTOR, a not-for-profit organization with a mission to help the scholarly community take advantage of advances in technology. For more information regarding JSTOR, please contact support@jstor.org. 


\title{
APPROXIMATION OF UPPER LYAPUNOV EXPONENTS OF BILINEAR STOCHASTIC DIFFERENTIAL SYSTEMS*
}

\author{
DENIS TALAY ${ }^{\dagger}$
}

\begin{abstract}
A bilinear Stochastic Differential System is considered whose solution starting from $x$ is denoted by $(X(t, x))$; it is supposed that its (upper) Lyapunov exponent $\lambda$ exists. The purpose of the paper is to propose an efficient algorithm to approximate $\lambda$.

It is shown that, for Markov chains $\left(\bar{X}_{p}^{h}(x), p \in \mathbb{N}\right)$ defined by several approximation schemes, the Lyapunov exponents $\bar{\lambda}^{h}$ are well defined and can be computed; estimates are given in terms of the discretization step $h$ of the theoretical error $\left|\lambda-\bar{\lambda}^{h}\right|$.

Then these results are applied to an engineering problem: the stability of the motion of helicopter rotor blades in a turbulent wind.
\end{abstract}

Key words. approximation of stochastic differential equations, Lyapunov exponents

AMS(MOS) subject classifications. 60H10, 65C20, 93E15, 93E25

1. Introduction. Let us consider a bilinear stochastic differential system in $\mathbb{R}^{d}$, written in the Stratonovich sense:

$$
d X(t)=A X(t) d t+\sum_{i=1}^{r} B_{i} X(t) \circ d W_{i}(t)
$$

where $\left\{W_{i}(t) ; 1 \leqq i \leqq r\right\}$ are mutually independent standard Wiener processes.

The solution starting at $x \in \mathbb{R}^{d}$ will be denoted by $(X(t, x))$.

Below we will state some hypotheses ensuring the following property of the process $(X(t))$ :

$$
\exists \lambda \in \mathbb{R}, \quad \forall x \in \mathbb{R}^{d}, \quad \lambda=\lim _{t \rightarrow+\infty} \frac{1}{t} \log |X(t, x)|, \quad \text { a.s. }
$$

The constant $\lambda$ is called the (upper) Lyapunov exponent of the system (1); the system is said to be stable if $\lambda$ is strictly negative: in that case, $|X(t, x)|$ decreases to zero exponentially fast.

In $\S 5$ we will discuss extensively an engineering problem, which has motivated this work: a study of stability for the motion of a helicopter rotor blade in turbulent winds. The mechanical model is a bilinear stochastic differential equation of dimension 4, whose coefficients depend on different parameters such as the velocity of the helicopter or some geometrical characteristics of the blade. The problem is to determine whether the turbulent winds may destabilize the system, for each interesting value of each parameter. In other terms, we are interested in the behaviour of the Lyapunov exponent with respect to the physical parameters of the system and the intensity of the noise.

But, except in some very particular cases, there exists no explicit formula that permits us to obtain the exact value of $\lambda$.

In the presence of a small noise, and when $(X(t))$ is a two-dimensional process, Auslender and Mil'shteĭn [3] and Pardoux and Wihstutz [22] have given asymptotic expansions of $\lambda$ in powers of the intensity of the noise.

\footnotetext{
* Received by the editors August 29 1988; accepted for publication (in revised form) August 21, 1990.

$\dagger$ Institut National de Recherche en Informatique et en Automatique, Avenue Emile Hugues, Sophia Antipolis, F-06565 Valbonne, France.
} 
To treat the general case, we propose to discretize in time the system (1), in order to get an approximate process that can easily be simulated on a computer, denoted by $\left(\bar{X}_{t}^{h}(x)\right)$ ( $h$ being the discretization step and $x$ the initial value), and to compute numerically for some large $T$ :

$$
\frac{1}{T} \log \left|\bar{X}_{T}^{h}(x)\right|
$$

Pardoux and Pignol [19] have proposed a discretization scheme and shown the convergence towards $\lambda$ of the almost sure limit:

$$
\lim _{p \rightarrow+\infty} \frac{1}{p h} \log \left|\bar{X}_{p h}^{h}(x)\right| .
$$

But the convergence rate was not estimated (under our hypotheses, our results below show that this method is of order $O(h)$ ).

Here we propose discretization methods such that

$$
\exists \bar{\lambda}^{h} \in \mathbb{R}, \quad \forall x \in \mathbb{R}^{d}-\{0\}: \bar{\lambda}^{h}=\lim _{p \rightarrow+\infty} \frac{1}{p h} \log \left|\bar{X}_{p h}^{h}(x)\right|, \quad \text { a.s. }
$$

and, moreover,

$$
\left|\lambda-\bar{\lambda}^{h}\right|=O(h) \quad \text { or } \quad O\left(h^{2}\right) .
$$

The organization of the paper is as follows. In $\S 2$, we review a fundamental theorem on the existence of $\lambda$. In $\S 3$, we present three discretization methods; in $\S 4$, we state our main result, which concerns the convergence rate of these schemes. In $\S 5$, we present the engineering problem of the helicopter rotor blades, the application of the previous results to solve that problem, and we discuss the obtained numerical results. In $\S 6$, we introduce a class of second-order schemes.

2. Lyapunov exponents. The purpose of this section is to introduce the material necessary for the sequel, essentially two theorems concerning the Lyapunov exponents of products of random matrices and of stochastic dynamical systems (we do not present them in their most general versions).

The theory of products of random matrices is presented in Bougerol and Lacroix [5] or Ledrappier [13].

The main aspects of the theory of Lyapunov exponents of stochastic dynamical systems are developed in the contributions to the workshop held in Bremen (1984): in particular, those of Arnold, Kliemann, and Oeljeklaus [1], and Arnold, Oeljeklaus, and Pardoux [2], which will be referred to often. A review of the theory and of recent results can also be found in Pardoux and Talay [21].

Let $S^{d-1}=\left\{x \in \mathbb{R}^{d} ;|x|=1\right\}$ be the unit sphere of $R^{d}$, and $P^{d-1}$ be the projective space of $R^{d}$, i.e., the quotient of $S^{d-1}$ with respect to the relation $u \sim v$ if and only if $u=-v$.

Let $G l\left(\mathbb{R}^{d}\right)$ be the set of invertible real $d \times d$ matrices. A given subset $\mathscr{S}$ of $G l\left(\mathbb{R}^{d}\right)$ is called irreducible if there does not exist a proper linear subspace of $\mathbb{R}^{d}, V$, such that

$$
\forall M \in \mathscr{S}, \quad M(V)=V .
$$

THEOREM 2.1 (Furstenberg). Let $\left(M_{n}\right)$ be a sequence of independent random matrices in $G l\left(\mathbb{R}^{d}\right)$ with common distribution $\nu$, such that (i) $E \log ^{+}\left|M_{1}\right|<+\infty$ and $E \log ^{+}\left|M_{1}^{-1}\right|<+\infty$.

(ii) The smallest subgroup of $G l\left(\mathbb{R}^{d}\right)$ containing the support of $\nu$ is irreducible. 
Then there exists a real number $\gamma$ such that, for any $x$ in $\mathbb{R}^{d}-\{0\}$,

$$
\gamma=\lim _{n \rightarrow+\infty} \frac{1}{n} \log \left|M_{n}, \cdots, M_{1} x\right|, \quad \text { a.s. }
$$

and for any $\nu$-invariant distribution $\mu$ on $P^{d-1}$,

$$
\gamma=\iint \log \frac{|M x|}{|x|} d \nu(M) d \mu(\bar{x})
$$

(where $\bar{x}$ is the class of $x$ in $P^{d-1}$ ).

For the continuous time case, we will use a result concerning a less general situation.

Let $s(t)$ be the process on $P^{d-1}$ defined as the equivalence class of $X(t) /|X(t)|$.

The process $s(t)$ is the solution of the following Stratonovich stochastic differential equation, describing a diffusion process in $P^{d-1}$ :

$$
d s(t)=h(A, s(t)) d t+\sum_{j=1}^{r} h\left(B_{j}, s(t)\right) \circ d W_{j}(t)
$$

where

$$
h(C, s):=C s-(C s, s) s .
$$

Let us introduce $\Lambda=L . A .\left\{h(A, \cdot), h\left(B_{1}, \cdot\right), \cdots, h\left(B_{k}, \cdot\right)\right\}$, i.e., the smallest vector space of differential operators containing the operators

$$
\sum_{i} h^{i}(A, \cdot) \partial_{i}, \quad \sum_{i} h^{i}\left(B_{j}, \cdot\right) \partial_{i} \quad(j=1, \cdots, r)
$$

and closed under the bracket operation $\left[P_{1}, P_{2}\right]=P_{1} \circ P_{2}-P_{2} \circ P_{1}$.

For $s$ in $P^{d-1}$, the space $\Lambda(s)$ will be the space obtained by considering all the elements of $\Lambda$ with all the coefficients of the operators frozen at their value in $s$.

In Arnold, Oeljeklaus, and Pardoux [2] is proved the following theorem (see also Bougerol and Lacroix [5]).

THEOREM 2.2. Let us suppose

$$
\operatorname{dim} \Lambda(s)=d-1 \quad \forall s \in P^{d-1} .
$$

Then the process $(s(t))$ on $P^{d-1}$ has a unique invariant probability measure $\mu$, and

(i) There exists a real number $\lambda$ such that, for any $x$ in $\mathbb{R}^{d}-\{0\}$,

$$
\lambda=\lim _{t \rightarrow+\infty} \frac{1}{t} \log |X(t, x)|, \quad \text { a.s. }
$$

(ii) Moreover, $\lambda$ satisfies

$$
\lambda=\int_{P^{d-1}} Q(s) d \mu(s)
$$

where

$$
Q(s):=(A s, s)+\frac{1}{2} \sum_{i=1}^{r}\left[\left(B_{i}^{2} s, s\right)+\left|B_{i} s\right|^{2}-2\left(B_{i} s, s\right)^{2}\right] .
$$

3. Discretization schemes. The rates of convergence of several discretization schemes have already been established, according to various criteria of convergence, including quadratic mean approximation (Clark and Cameron [6], Mil'shteǐn [14], Platen [24], Rumelin [25]), pathwise approximation (Talay [26]), approximation of 
expectations of the solution (Mil'shteĭn [15], Mil'shteǐn [16], Talay [27], Talay [28]), asymptotical efficiency for the minimization of the normalized quadratic mean error (Clark [7], Newton [17]), and approximation of the invariant probability measure in the ergodic case (Talay [29]).

A review of the main results concerning the first three points can be found in Pardoux and Talay [20].

In this section, we will consider three particular schemes, applied to the bilinear system (1). In $\S 6$ we will explain why these schemes have been chosen, and we will study a larger family of schemes.

\subsection{Notation and definitions.}

- $I$ will be the $d \times d$ identity matrix.

- $\tilde{A}$ will be the matrix $A+\frac{1}{2} \sum_{i=1}^{r} B_{i}^{2}$.

- For any matrix $\sigma, \sigma_{j}$ will denote the $j$ th column of $\sigma$; if $\sigma\left(x_{1}, \cdots, x_{d}\right)$ is a matrix-valued application, $\partial \sigma_{j}$ denotes the matrix-valued application whose element of the $i$ th row and $k$ th column is $\partial_{k} \sigma_{j}^{i}$.

The discretization schemes below will depend on three families of random variables: $\left(U_{p+1}^{j}\right),\left(Z_{p+1}^{k j}\right)$, and $\left(S_{p+1}^{k j}\right)$. The hypotheses concerning the family $\left(U_{p+1}^{j}\right)$ will be stated later. Moreover, in all the sequel, the sequence

$$
\left(U_{p+1}^{j}, Z_{p+1}^{k j}, j, k=1, \cdots, r, p \in \mathbb{N}\right)
$$

will be a family of independent random variables; the $\left(Z_{p+1}^{k j}\right)$ are independently and identically distributed, their common law being defined by

$$
P\left(Z_{p}^{k j}=\frac{1}{2}\right)=P\left(Z_{p}^{k j}=-\frac{1}{2}\right)=\frac{1}{2} ;
$$

the family $\left(S_{p}^{k j}\right)$ is defined by

$$
\begin{array}{ll}
S_{p+1}^{k j}=\frac{1}{2} U_{p+1}^{k} U_{p+1}^{j}+Z_{p+1}^{k j}, & k<j, \\
S_{p+1}^{k j}=\frac{1}{2} U_{p+1}^{k} U_{p+1}^{j}-Z_{p+1}^{j k}, & k>j, \\
S_{p+1}^{j j}=\frac{1}{2}\left[\left(U_{p+1}^{j}\right)^{2}-1\right] . &
\end{array}
$$

3.2. Three discretization schemes. Let us consider the schemes defined by

$$
\bar{X}_{p+1}^{h}=M_{p+1}^{h} \bar{X}_{p}^{h}
$$

with

$$
M_{p+1}^{h}=I+\sum_{j=1}^{r} B_{j} U_{p+1}^{j} \sqrt{h}+\tilde{A} h
$$

or

$$
M_{p+1}^{h}=I+\sum_{j=1}^{r} B_{j} U_{p+1}^{j} \sqrt{h}+A h+\frac{1}{2} \sum_{j=1}^{r} B_{j}^{2}\left(U_{p+1}^{j}\right)^{2} h+\sum_{\substack{j, k=1 \\ j \neq k}}^{r} B_{j} B_{k} S_{p+1}^{k j} h
$$

or

$$
\begin{aligned}
M_{p+1}^{h}=I & +\sum_{j=1}^{r} B_{j} U_{p+1}^{j} \sqrt{h}+A h+\frac{1}{2} \sum_{j=1}^{r} B_{j}^{2}\left(U_{p+1}^{j}\right)^{2} h+\sum_{\substack{j, k=1 \\
j \neq k}}^{r} B_{j} B_{k} S_{p+1}^{k j} h \\
& +\frac{1}{2} \sum_{j=1}^{r}\left(\tilde{A} B_{j}+B_{j} \tilde{A}\right) U_{p+1}^{j} h^{3 / 2}+\frac{1}{2} \tilde{A}^{2} h^{2} .
\end{aligned}
$$


These schemes will be tested numerically in $\S 5.3$. The first one will be called the Euler scheme, the second one the Mil'shteĭn scheme.

Except in $\S 6,\left(\bar{X}_{p}^{h}(x)\right)$ will denote the Markov chain defined by one of these schemes, with initial value $x$.

3.3. Example: The one-dimensional case. Let us consider the elementary case $d=r=1$.

The system is written again under the following form (with $a$ and $b$ scalar constants):

$$
d X(t)=a X(t) d t+b X(t) \circ d W(t) .
$$

The solution is $X(t)=X(0) \exp (a t+b W(t))$, thus $\lambda=a$.

With the particular choice $\sqrt{h} U_{p+1}^{j}=W((p+1) h)-W(p h)$, denoted by $\Delta_{p+1}^{h} W$, the Mil'shteĭn scheme becomes

$$
\bar{X}_{p+1}^{h}=\left(1+b \Delta_{p+1}^{h} W+a h+\frac{1}{2} b^{2}\left(\Delta_{p+1}^{h} W\right)^{2}\right) \bar{X}_{p}^{h} .
$$

The strong law of large numbers implies the existence of $\bar{\lambda}^{h}$ (defined as in (2)). Moreover, it can easily be checked that for any nonzero initial condition

$$
\exists C \in \mathbb{R}, \frac{1}{p^{2} h^{2}} E\left(\log \left|\bar{X}_{p}^{h}(x)\right|\right)^{2}<C,
$$

so that the sequence $\left(1 / p h \log \left|\bar{X}_{p}^{h}(x)\right|\right)$ is uniformly integrable, and therefore $\bar{\lambda}^{h}$ satisfies

$$
\bar{\lambda}^{h}=\lim _{p \rightarrow+\infty} \frac{1}{p h} E \log \left|\bar{X}_{p}^{h}(x)\right| \quad \forall x \in \mathbb{R}-\{0\} .
$$

But, for $h$ small enough,

$$
\begin{aligned}
E \log \left|\bar{X}_{p}^{h}(x)\right|-\log |x|= & p E \log \left|1+b \Delta_{1}^{h}+a h+\frac{1}{2} b^{2}\left(\Delta_{1}^{h}\right)^{2}\right| \\
= & \frac{p}{\sqrt{2 \pi h}} \int_{\mathbb{B}} \log \left|1+b y+a h+\frac{1}{2} b^{2} y^{2}\right| \exp \left(-\frac{y^{2}}{2 h}\right) d y \\
= & \frac{p}{\sqrt{2 \pi}} \int_{0}^{+\infty} \log \left(1-b y \sqrt{h}+a h+\frac{1}{2} b^{2} y^{2} h\right) \exp \left(-\frac{y^{2}}{2}\right) d y \\
& +\frac{p}{\sqrt{2 \pi}} \int_{0}^{+\infty} \log \left(1+b y \sqrt{h}+a h+\frac{1}{2} b^{2} y^{2} h\right) \exp \left(-\frac{y^{2}}{2}\right) d y \\
= & \frac{p}{\sqrt{2 \pi}} \int_{0}^{+\infty} \log \left(1+2 a h+\left(a+\frac{1}{2} b^{2} y^{2}\right)^{2} h^{2}\right) \exp \left(-\frac{y^{2}}{2}\right) d y \\
= & p a h+p O\left(h^{2}\right) .
\end{aligned}
$$

Therefore (and the same holds for the Euler scheme (6)), we have

$$
\bar{\lambda}^{h}=a+O(h)=\lambda+O(h) .
$$

An analogous computation for the scheme (8) would lead to

$$
\bar{\lambda}^{h}=a+O\left(h^{2}\right)=\lambda+O\left(h^{2}\right) .
$$

4. Theoretical results. In this section, our aim is to prove that the Euler scheme (6) and the Mil'shteĭn scheme (7) are of first order for the approximation of the Lyapunov exponents, whereas the scheme (8) is of second order.

\subsection{Hypotheses.}

Hypothesis (H0). The system (3) can be viewed as describing a diffusion on $S^{d-1}$.

We will suppose the following hypothesis. 
(H0) The infinitesimal generator $\mathscr{L}$ of the process $(s(t))$ on $S^{d-1}$ is uniformly elliptic, i.e., there exists a strictly positive constant $\alpha$ such that, for any $x$ in $S^{d-1}$ and any vector $\xi$ in the tangent space $T_{S^{d-1}}(x)$,

$$
\sum_{i=1}^{r}\left(h\left(B_{i}, x\right), \xi\right)^{2} \geqq \alpha|\xi|^{2} .
$$

This condition is more restrictive than $(\mathrm{H})$. But, if $(\mathrm{H})$ is sufficient to ensure the existence of the Lyapunov exponents $\bar{\lambda}^{h}$ of the Markov chains defined by the considered schemes (see below), it seems difficult not to use (H0) to get estimations of the approximation error $\lambda-\bar{\lambda}^{h}$. Let us show a very simple example of a situation where (H0) is fulfilled: $r=1$ and

$$
B=\left[\begin{array}{rr}
0 & -1 \\
1 & 0
\end{array}\right]
$$

Hypothesis (HU). For our proofs, we need a technical hypothesis on the random variables $U_{p+1}^{j}$ 's involved by the discretization schemes, which is not limitative from a practical point of view; (ii) was unnecessary for the theoretical results concerning the approximation of the law of diffusion processes (cf. the Appendix ( $\$ 6$ )).

(HU)(i) The $\left(U_{p+1}^{j}\right)$ 's are independently and identically distributed, and the following conditions on the moments are fulfilled:

$$
\begin{aligned}
E\left[U_{p+1}^{j}\right]=E\left[U_{p+1}^{j}\right]^{3}= & E\left[U_{p+1}^{j}\right]^{5}=0, \\
& E\left[U_{p+1}^{j}\right]^{2}=1, \\
& E\left[U_{p+1}^{j}\right]^{4}=3, \\
& E\left[U_{p+1}^{j}\right]^{n}<+\infty \quad \forall n>5 .
\end{aligned}
$$

(ii) The common law of the ( $\left.U_{p+1}^{j}\right)$ 's has a continuous density with respect to the Lebesgue measure; the support of this density contains an open interval including zero and is compact.

4.2. Existence of the Lyapunov exponent. Let us begin by showing the existence of the upper Lyapunov exponent for the Markov chains defined by the discretization schemes.

Proposition 4.1. Let us suppose that the system (3) satisfies $(\mathrm{H})$.

Let $\left(\bar{X}_{p}^{h}, p \in \mathbb{N}\right)$ be defined by the Euler scheme (6), the Mil'shtein scheme (7), or scheme (8).

Then, under (HU)(i), (i) for any $h$ small enough, there exists a real number $\bar{\lambda}^{h}$ satisfying

$$
\forall x \in \mathbb{R}^{d}-\{0\}: \bar{\lambda}^{h}=\lim _{p \rightarrow+\infty} \frac{1}{p h} \log \left|\bar{X}_{p}^{h}(x)\right|, \quad \text { a.s. }
$$

(ii) Moreover,

$$
\forall x \in \mathbb{R}^{d}-\{0\}: \bar{\lambda}^{h}=\lim _{p \rightarrow+\infty} \frac{1}{p h} E \log \left|\bar{X}_{p}^{h}(x)\right| .
$$

Proof of Proposition 4.1. Let us prove (i).

Our aim is to apply the Furstenberg Theorem 2.1.

Let us suppose the existence of a stepsize $h$ such that the smallest semigroup of $G l\left(\mathbb{R}^{d}\right)$ containing the support of the law of $M_{1}^{h}$ is not irreducible. Then, there would exist a proper linear subspace of $\mathbb{R}^{d}, V^{h}$, such that

$$
M_{1}^{h}\left(V^{h}\right)=V^{h} \text {, a.s. }
$$


Let $x$ any vector of $V^{h}$. Then, for all $j, E\left[U_{1}^{j} M_{1}^{h} x\right]$ and $E\left[M_{1}^{h} x\right]$ would belong to $V^{h}$; therefore we would have $B_{j}\left(V^{h}\right) \subset V^{h}$ and $A\left(V^{h}\right) \subset V^{h}$.

In that case, system (3) with an initial condition $x$ belonging to $V^{h}$ can be viewed as a system on $V^{h}$, and for any time $t, X(t, x)$ would belong to $V^{h}$.

This assertion cannot be true under Hypothesis $(\mathrm{H})$, since in that case (see $\S 1$ of [2]) the semigroup of $(s(t))$ leaves no submanifold of dimension less than $d-1$ in $P^{d-1}$ invariant.

Finally, all the hypotheses of Theorem 2.1 are fulfilled, and (i) is proved for the Mil'shteĭn scheme.

Now, let us consider scheme (8).

Let us suppose that, for all stepsize $h_{0}$, there exists $h<h_{0}$ such that the smallest semigroup of $G l\left(\mathbb{R}^{d}\right)$ containing the support of the law of $M_{1}^{h}$ is not irreducible, and let us prove that, in that case, there would exist a proper linear subspace $V$ such that

$$
A(V) \subset V \quad \text { and } \quad B_{j}(V) \subset V \quad(j=1, \cdots, r)
$$

(as mentioned, this property contradicts our hypotheses).

Thus, for any integer $n$, let us choose $h_{n}<1 / n$ and $V^{n}$ a proper linear subspace satisfying

$$
M_{1}^{h_{n}}\left(V^{n}\right)=V^{n}, \quad \text { a.s. }
$$

Let $x$ any vector of $V^{n}$. Then, for all $j, E\left[\left(U_{1}^{j}\right)^{2} M_{1}^{h_{n}} x\right], E\left[\left(U_{1}^{j}\right) M_{1}^{h_{n}} x\right]$ and $E\left[M_{1}^{h_{n}} x\right]$ would belong to $V^{n}$; therefore we would have

$$
\begin{aligned}
& B_{j}^{2}\left(V_{n}\right) \subset V_{n}, \\
& \left(A+\frac{1}{2} \tilde{A}^{2} h_{n}\right)\left(V_{n}\right) \subset V_{n}, \\
& \left(B_{j}+\frac{1}{2}\left(\tilde{A} B_{j}+B_{j} \tilde{A}\right) h_{n}\right)\left(V_{n}\right) \subset V_{n} .
\end{aligned}
$$

Let us define

$$
l=\lim _{n} \inf \operatorname{dim}\left(V_{n}\right)
$$

and $\left(m_{n}\right)$ the subsequence of indices such that $V_{m_{n}}$ is $l$-dimensional.

For each $V_{m_{n}}$, we choose a basis of unit-normed and orthogonal vectors $\left(e_{m_{n}}^{k}\right.$, $k=1, \cdots, l)$.

We can choose a new subsequence (again denoted $\left.\left(m_{n}\right)\right)$ such that $\left(\left(e_{m_{n}}^{k}, k=\right.\right.$ $1, \cdots, l), n \in \mathbb{N})$ converges for the strong topology on $\mathbb{R}^{l}$; let $\left(u^{k}, k=1, \cdots, l\right)$ be the limit.

Let us fix $k$ in $\{1, \cdots, l\}$; we may write (as a consequence of (11))

$$
\left(A+\frac{1}{2} \tilde{A}^{2} h_{m_{n}}\right) e_{m_{n}}^{k}=\sum_{j=1}^{l} \alpha_{m_{n}}^{j}(k) e_{m_{n}}^{j} .
$$

Now, for each $j=1, \cdots, l$, the previous equality implies

$$
\left(\left(A+\frac{1}{2} \tilde{A}^{2} h_{m_{n}}\right) e_{m_{n}}^{k}, e_{m_{n}}^{j}\right)=\alpha_{m_{n}}^{j}(k),
$$

so that the sequence $\left(\alpha_{m_{n}}^{j}(k), n \in \mathbb{N}\right)$ converges to $\left(A u^{k}, u^{j}\right)$, and therefore

$$
A u^{k}=\sum_{j=1}^{l}\left(A u^{k}, u^{j}\right) u^{j}
$$

$V$ being the subspace generated by $\left(u^{k}, k=1, \cdots, l\right)$, we have just proved (10) for $A$; but similar arguments and (11) permit us to obtain the whole result for scheme (8). Similar arguments can be used for the Euler scheme. 
To prove (ii), it just remains to check that the sequence $\left(1 / p h \log \left|\bar{X}_{p}^{h}(x)\right|, p \in \mathbb{N}\right)$ is uniformly integrable.

Actually, using the inequality $\left|a_{1}+\cdots+a_{p}\right|^{2} \leqq p\left(a_{1}^{2}+\cdots+a_{p}^{2}\right)$, we obtain

$$
\begin{aligned}
\frac{1}{p^{2} h^{2}} E\left(\log \left|\bar{X}_{p}^{h}(x)\right|\right)^{2} & \leqq \frac{1}{p^{2} h^{2}} E\left(\log \prod_{j=1}^{p}\left|M_{j}^{h}\right|+\log |x|\right)^{2} \\
& \leqq \frac{2}{h^{2}} E\left(\log \left|M_{1}^{h}\right|\right)^{2}+\frac{2}{p^{2} h^{2}}(\log |x|)^{2} .
\end{aligned}
$$

This ends the proof.

4.3. Convergence rates. Let us give estimates for $\left|\lambda-\bar{\lambda}^{h}\right|$.

THEOREM 4.2. Let us suppose that system (3) satisfies Hypothesis (H0).

Let $\left(\bar{X}_{p}^{h}, p \in \mathbb{N}\right)$ be defined by either the Euler scheme (6), the Mil'shtein scheme (7), or scheme (8), and let us suppose (HU).

Then, if $\lambda$ is the upper Lyapunov exponent of (1): (i) for $\left(\bar{X}_{p}^{h}\right)$ defined by the Euler or Mil'shtěn scheme, $\left|\lambda-\bar{\lambda}^{h}\right|=O(h)$;

(ii) for $\left(\bar{X}_{p}^{h}\right)$ defined by scheme $\left.(8), \mid \lambda-\bar{\lambda}^{h}\right)=O\left(h^{2}\right)$.

4.3.1. Remark. Under (HU), the process $\left(\bar{s}_{p}^{h}\right)$ defined on $S^{d-1}$ by

$$
\bar{s}_{p}^{h}=\frac{\bar{X}_{p}^{h}}{\left|\bar{X}_{p}^{h}\right|}
$$

is ergodic; let $\bar{\mu}^{h}$ be its unique invariant probability measure; if the function $\bar{Q}^{h}$ is defined by

$$
\bar{Q}^{h}(s)=\frac{1}{h} E \log \left|\bar{X}_{1}^{h}(s)\right|,
$$

we can write (cf. $\S 4.3 .3$ and the Furstenberg Theorem 2.1)

$$
\bar{\lambda}^{h}=\int_{S^{d-1}} \bar{Q}^{h}(s) d \bar{\mu}^{h}(s)
$$

so that

$$
\lambda-\bar{\lambda}^{h}=\int_{S^{d-1}} Q(s)\left(d \mu(s)-d \bar{\mu}^{h}(s)\right)+\int_{S^{d-1}}\left(Q(s)-\bar{Q}^{h}(s)\right) d \bar{\mu}^{h}(s) .
$$

We will see below that, for any smooth function on $S^{d-1}$, scheme (8) satisfies

$$
\int_{S^{d-1}} f(s)\left(d \mu(s)-d \bar{\mu}^{h}(s)\right)=O\left(h^{2}\right) .
$$

But

$$
Q(s)-\bar{Q}^{h}(s)=O(h)
$$

Therefore, more accurate estimations are needed. In fact, the result to be proved below shows that

$$
\int_{S^{d-1}}\left(Q(s)-\bar{Q}^{h}(s)\right) d \mu(s)=O\left(h^{2}\right) .
$$

4.3.2. Two lemmas. Let $n \in \mathbb{N}^{*}$ and $x \in S^{d-1}$ be fixed. The antipodal point of $x$ is denoted by $x^{*}$. 
Let $U_{x}$ be the set of points of $S^{d-1}$ whose geodesic distance from $x$ is less than $4 \pi / 3\left(2 \pi\right.$ is the maximal geodesic distance on $\left.S^{d-1}\right)$.

Let $y \in U_{x} \rightarrow \phi_{x}(y)$ the stereographic projection of pole $x^{*}$.

LEMMA 4.3. Let us suppose that Hypothesis ( $\mathrm{H} 0)$ is satisfied.

Let $(s(t, \cdot))$ define the stochastic flow of equation (3). by

Let $f$ be a real $C^{\infty}$ function on $S^{d-1}$, and let us define the function $u(t, y)$ on $\mathbb{R}_{+} \times S^{d-1}$

$$
u(t, y)=E f(s(t, y)) .
$$

Let $\mu$ be the unique invariant probability measure of the process $(s(t))$. Then there exist strictly positive constants $\Gamma$ and $\gamma$ such that

$$
\forall x \in S^{d-1}:\left|u(t, x)-\int_{S^{d-1}} f(s) d \mu(s)\right| \leqq \Gamma \exp (-\gamma t)
$$

and, for any multi-index $I$, there exist strictly positive constants $\Gamma_{I}$ and $\gamma_{I}$ such that, for any $x$ in $S^{d-1}$, any $\theta$ in $\phi_{x}\left(U_{x}\right)$, the spatial derivative $\partial_{I} u\left(t, \phi_{x}^{-1}(\theta)\right)$ satisfies

$$
\left|\partial_{I} u\left(t, \phi_{x}^{-1}(\theta)\right)\right| \leqq \Gamma_{I} \exp \left(-\gamma_{I} t\right) .
$$

Proof of Lemma 4.3. In Talay [29], a result similar to (13) was established for a diffusion process in the whole space, under some suitable conditions on the coefficients of the system; here, instead of these conditions, we will use the fact that $S^{d-1}$ is a compact manifold.

We begin by proving (12). The condition ( $\mathrm{H} 0)$ implies the existence of a continuous and strictly positive density for the transition probability of the process $(s(t))$; therefore, since $S^{d-1}$ is compact, $(s(t))$ satisfies the so-called Doeblin condition: there exist $\delta>0$, $\varepsilon>0, t>0$, such that, for all open set $E$ of $S^{d-1}$ with Lebesgue measure less than $\delta: P(t, y, E) \leqq 1-\varepsilon$, uniformly in $y$. Consequently, the inequality (12) is satisfied (see Doob [8, p. 193], e.g.).

Now, let us prove the second part of the lemma.

Let us introduce the differential operators on $S^{d-1}$ defined by

$$
\mathscr{L}_{j}=\sum_{i=1}^{d} h^{i}\left(B_{j}, \cdot\right) \partial_{i}
$$

and let $\mathscr{L}$ be the infinitesimal generator of the process $(s(t))$ solution of (3) on $S^{d-1}$.

It is well known that

$$
\begin{aligned}
& \frac{d}{d t} u(t, y)=\mathscr{L} u(t, y), \\
& u(0, y)=f(y),
\end{aligned}
$$

so that we deduce that

$$
\frac{d}{d t}|u(t, y)|^{2}-\mathscr{L}|u(t, y)|^{2}=-\sum_{k=1}^{r}\left(\mathscr{L}_{k} u(t, y)\right)^{2} .
$$

We multiply the previous equality by $e^{\delta_{0} t}$, we integrate with respect to $\mu$, and then we integrate from zero to $T$ ( $T$ being an arbitrarily large time). Using $\mathscr{L}^{*} \mu=0$, we deduce that, for any $0<\delta_{0}<\gamma$,

$$
\sum_{k=1}^{r} \int_{0}^{+\infty} e^{\delta_{0} t} \int_{S^{d-1}}\left|\mathscr{L}_{k} u(t, y)\right|^{2} d \mu d t<+\infty
$$

(a similar computation is performed in Talay $[29, \S 6.1 .2]$ ). 
We may also check that there exist strictly positive constants $C_{1}$ and $C_{2}$ such that

$$
\begin{aligned}
\frac{d}{d t}\left(\sum_{k=1}^{r}\left|\mathscr{L}_{k} u(t, y)\right|^{2}\right) & -\mathscr{L}\left(\sum_{k=1}^{r}\left|\mathscr{L}_{k} u(t, y)\right|^{2}\right) \\
& \leqq-C_{1} \sum_{k, l=1}^{r}\left|\mathscr{L}_{k} \mathscr{L}_{l} u(t, y)\right|^{2}+C_{2} \sum_{k=1}^{r}\left|\mathscr{L}_{k} u(t, y)\right|^{2}
\end{aligned}
$$

Proceeding as just above, we obtain for any strictly positive $\delta_{1}<\delta_{0}$

$$
\sum_{k=1}^{r} \int_{S^{d-1}}\left|\mathscr{L}_{k} u(t, y)\right|^{2} d \mu \leqq C e^{-\delta_{1} t} .
$$

Let $d \tau$ be the Riemannian measure on $S^{d-1}$ induced by the tensor field $\left(a_{i j}\right)^{-1}$, where $a$ is the matrix $\sigma \sigma^{*}$, with $\sigma(s)=\sum_{j=1}^{r} h\left(B_{j}, s\right)$.

Hypothesis ( $\mathrm{H} 0)$ implies that $\mu$, the invariant probability measure of the process $(s(t))$, has a smooth density $p(\cdot)$ with respect to $d \tau$, which solves $\mathscr{L}^{*} p=0$, and is strictly positive (cf. Ikeda and Watanabe [10, Chap. V]); therefore, we can bound it from below on $S^{d-1}$ by a strictly positive constant.

From this remark, we deduce from the previous inequality the existence of strictly positive constants $C, \alpha$, independent on $x$, such that

$$
\int_{\phi_{x}\left(U_{x}\right)}\left|\nabla u\left(t, \phi_{x}^{-1}(\theta)\right)\right|^{2} d \theta \leqq C e^{-\alpha t} .
$$

By the same procedure, it may be shown that, for any sequence $J=\left(k_{1}, \cdots, k_{q}\right)$ of integers in $1, \cdots, r$, there exist strictly positive constants $C_{J}$ and $\alpha_{J}$ such that

$$
\int_{S^{d-1}}\left|\mathscr{L}_{k_{1}} \cdots \mathscr{L}_{k_{q}} u(t, y)\right|^{2} d \mu \leqq C_{J} \exp \left(-\alpha_{J} t\right) .
$$

Note the existence of a constant $C_{n}$, independent on $x$, such that ( $\tilde{b}$ and $\tilde{\sigma}$ being the coefficients of $\mathscr{L}$ in the local coordinates $\theta$ )

$$
\begin{array}{r}
\forall \theta \in \phi_{x}\left(U_{x}\right):|\tilde{b}(\theta)| \leqq C_{n}, \\
\left|\tilde{\sigma}_{i j}(\theta)\right| \leqq C_{n},
\end{array}
$$

and for all multi-index $I$ of length less than $n$,

$$
\begin{aligned}
& \forall \theta \in \phi_{x}\left(U_{x}\right):\left|\partial_{I} \tilde{b}(\theta)\right| \leqq C_{n}, \\
& \left|\partial_{I} \tilde{\sigma}_{i j}(\theta)\right| \leqq C_{n} .
\end{aligned}
$$

We deduce that for any multi-index $J$ (referring to derivatives with respect to the coordinates $\theta_{1}, \cdots, \theta_{d-1}$ ), there exist strictly positive constants $C_{J}$ and $\alpha_{J}$, independent on $x$, such that

$$
\int_{\phi_{x}\left(U_{x}\right)}\left|\partial_{J} u\left(t, \phi_{x}^{-1}(\theta)\right)\right|^{2} d \theta \leqq C_{J} \exp \left(-\alpha_{J} t\right) .
$$

We conclude by applying the Sobolev Imbedding Theorem.

LEMMA 4.4. Let us suppose that Hypothesis (H0) is satisfied, and let $\mu$ be the unique invariant probability measure of $(s(t))$.

Let us define the Markov chain $\left(\bar{s}_{p}^{h}\right)$ on $S^{d-1}$ by

$$
\bar{s}_{p}^{h}=\frac{\bar{X}_{p}^{h}}{\left|\bar{X}_{p}^{h}\right|}
$$


where $\left(\bar{X}_{p}^{h}\right)$ is defined by the Euler scheme (6) or the Mil'shtein scheme (7), under condition (HU).

Then, for any $x$ in $\mathbb{R}^{d}-\{0\}$, for any smooth function $f: S^{d-1} \rightarrow \mathbb{R}$,

$$
\left|\int_{S^{d-1}} f(\theta) d \mu(\theta)-\lim _{N \rightarrow+\infty} \frac{1}{N} \sum_{p=1}^{N} f\left(\bar{s}_{p}^{h}\left(\frac{x}{|x|}\right)\right)\right|=O(h), \quad \text { a.s. }
$$

The above approximation error is of order $O\left(h^{2}\right)$ for scheme (8).

Proof of Lemma 4.4. We will follow Talay [29, § 7.2], and only consider scheme (8), the other case being simpler.

First, we define the symbol $\stackrel{E}{=}$ : we will write $X \stackrel{E}{=} Y$ instead of $E(X)=E(Y)$. We will also write $\tilde{x}$ for $x /|x|$.

Under (HU), for any $h$ small enough, almost surely $\bar{s}_{p+1}^{h}(\tilde{x})$ takes its value in $U_{\bar{s}_{p}^{h}(\tilde{x})}$.

With computations analogous to those of Talay [28], and performing a Taylor expansion up to order 6 of the function $\theta \rightarrow u\left(t, \phi_{\bar{s}_{p}^{h}(\tilde{x})}^{-1}(\theta)\right)$, we may check that

$$
u\left(j h, \bar{s}_{p+1}^{h}(\tilde{x})\right) \stackrel{E}{=} u\left(j h, \bar{s}_{p}^{h}(\tilde{x})\right)+\mathscr{L} u\left(j h, \bar{s}_{p}^{h}(\tilde{x})\right) h+\frac{1}{2} \mathscr{L}^{2} u\left(j h, \bar{s}_{p}^{h}(\tilde{x})\right) h^{2}+r_{j, p+1}^{h} h^{3}
$$

with the remainder term $r_{j, p+1}^{h}$ expressed as a sum of terms, each one being of the following form:

$$
\text { Constant } \times E\left[\psi_{I}(\gamma) \partial_{I} u\left(j h, \phi_{\bar{s}_{p}^{1}(\tilde{x})}^{-1}(\delta)\right)\right]
$$

where

- $\psi_{I}$ is a continuous function of the coordinates $\theta_{1}, \cdots, \theta_{d-1}$, then is bounded in $\phi_{\bar{s}_{p}^{h}(\tilde{x})}\left(U_{\bar{s}_{p}^{h}(\tilde{x})}\right)$,

- $\delta$ and $\gamma$ are in $\phi_{\bar{s}_{p}^{h}(\tilde{x})}\left(U_{\bar{s}_{p}^{h}(\tilde{x})}\right)$.

Thus, by using (13), it can be checked that the above remainder term satisfies for some strictly positive constants $C_{0}, C$, and $\gamma$ :

$$
\exists \gamma>0, \quad \sum_{j=0}^{+\infty}\left|r_{j, p+1}^{h}\right| \leqq \frac{C_{0}}{1-e^{-\gamma h}} \leqq \frac{C}{h} .
$$

Now we use equation (14) to write

$$
u\left((j+1) h, \bar{s}_{p}^{h}(\tilde{x})\right) \stackrel{E}{=} u\left(j h, \bar{s}_{p}^{h}(\tilde{x})\right)+\mathscr{L} u\left(j h, \bar{s}_{p}^{h}(\tilde{x})\right) h+\frac{1}{2} \mathscr{L}^{2} u\left(j h, \bar{s}_{p}^{h}(\tilde{x})\right) h^{2}+\tilde{r}_{j, p+1}^{h} h^{3}
$$

with a remainder term $\tilde{r}_{j, p+1}^{h}$ which can be expressed in the same manner as $r_{j, p+1}^{h}$.

Therefore, if we define $R_{j, p+1}^{h}$ by

$$
R_{j, p+1}^{h}=r_{j, p+1}^{h}-\tilde{r}_{j, p+1}^{h},
$$

$R_{j, p+1}^{h}$ satisfies, for some positive constant $C_{1}$,

$$
\sum_{j=0}^{+\infty}\left|R_{j, p+1}^{h}\right| \leqq \frac{C_{1}}{h} .
$$

Moreover,

$$
u\left(j h, \bar{s}_{p+1}^{h}(\tilde{x})\right) \stackrel{E}{=} u\left((j+1) h, \bar{s}_{p}^{h}(\tilde{x})\right)+R_{j, p+1}^{h} h^{3} .
$$

Remarking that

$$
\frac{1}{N} \sum_{p=1}^{N} f\left(\bar{s}_{p}^{h}(\tilde{x})\right)=\frac{1}{N} \sum_{p=1}^{N} u\left(0, \bar{s}_{p}^{h}(\tilde{x})\right)
$$


with successive uses of (17) we obtain

$$
\frac{1}{N} \sum_{p=1}^{N} f\left(\bar{s}_{p}^{h}(\tilde{x})\right) \stackrel{E}{=} \frac{1}{N} \sum_{p=1}^{N} u(p h, \tilde{x})+\frac{1}{N} \sum_{p=1}^{N} \sum_{j=0}^{p-1} R_{j, p}^{h} h^{3} .
$$

However, we note the following.

- Under Hypothesis (H0), the process $(s(t))$ is ergodic and $u(t, \tilde{x})$ satisfies

$$
u(t, \tilde{x})=E f(s(t, \tilde{x})) \text {. }
$$

Thus

$$
\lim _{N \rightarrow \infty} \frac{1}{N} \sum_{p=1}^{N} u(p h, \tilde{x})=\int_{S^{d-1}} f(s) d \mu(s)
$$

- The estimation (16) implies

$$
\frac{1}{N} \sum_{p=0}^{N} \sum_{j=0}^{p-1} R_{j, p}^{h} h^{3} \leqq C_{1} h^{2} .
$$

- Because the process $\left(\bar{s}_{p}^{h}\right)$ is ergodic,

$$
\lim _{N \rightarrow \infty} E \frac{1}{N} \sum_{p=1}^{N} f\left(\bar{s}_{p}^{h}(\tilde{x})\right)=\int_{S^{d-1}} f(s) d \bar{\mu}^{h}(s)=\text { a.s. } \lim _{N \rightarrow \infty} \frac{1}{N} \sum_{p=1}^{N} f\left(\bar{s}_{p}^{h}(\tilde{x})\right) .
$$

This ends the proof.

Proof of Theorem 4.2. Again, we only treat scheme (8).

We have previously shown that

$$
\bar{\lambda}^{h}=\lim _{N \rightarrow \infty} \frac{1}{N h} E \log \left|\bar{X}_{N}^{h}\right|
$$

and therefore

$$
\bar{\lambda}^{h}=\lim _{N \rightarrow \infty} \frac{1}{N h} \sum_{p=1}^{N} E \log \left|M_{p+1}^{h} \bar{s}_{p}^{h}\right| .
$$

Elementary (but long!) computations show that the scheme $\left(\bar{X}_{p}^{h}\right)$ satisfies, for any smooth function $f$ :

$$
E f\left(\bar{X}_{p+1}^{h}\right)=E f\left(\bar{X}_{p}^{h}\right)+E L f\left(\bar{X}_{p}^{h}\right) h+\frac{1}{2} E L^{2} f\left(\bar{X}_{p}^{h}\right) h^{2}+E \psi\left(\bar{X}_{p}^{h}+\theta\left(\bar{X}_{p+1}^{h}-\bar{X}_{p}^{h}\right)\right) h^{3}
$$

where $\psi$ is a function which can be expressed as a sum whose each term is a product of polynomial functions (with respect to the Euclidian coordinates) and of derivatives of the function $f$, and $\theta$ is in $] 0,1[$.

Under (HU), for any integer $n$, there exists a strictly positive constant $C_{n}$ such that

$$
\left|x+\theta\left(M_{1}^{h}-I\right) x\right|^{-n} \leqq C_{n}
$$

uniformly in $h$ small enough, in $\theta$ in $[0,1]$ and in $x$ in $S^{d-1}$.

Therefore, applying the previous remark to $f(x)=\log |x|$, if $Q$ is the function defined in (4), we obtain

$$
E \log \left|M_{p+1}^{h} \bar{s}_{p}^{h}\right|=E Q\left(\bar{s}_{p}^{h}\right) h+\frac{1}{2} E \mathscr{L} Q\left(\bar{s}_{p}^{h}\right) h^{2}+R_{p+1}^{h} h^{3}
$$

with $R_{p+1}^{h}$ uniformly bounded in $p \in \mathbb{N}$ and in $h$ small enough.

We deduce

$$
\frac{1}{N h} \sum_{p=1}^{N} E \log \left|M_{p+1}^{h} \bar{s}_{p}^{h}\right|=\frac{1}{N} \sum_{p=1}^{N} E Q\left(\bar{s}_{p}^{h}\right)+\frac{1}{2 N} \sum_{p=1}^{N} E \mathscr{L} Q\left(\bar{s}_{p}^{h}\right) h+\frac{1}{N} \sum_{p=1}^{N} R_{p+1}^{h} h^{2} .
$$


Using the result of the previous lemma, we deduce

$$
\bar{\lambda}^{h}=\int_{S^{d-1}} Q(s) d \mu(s)+\int_{S^{d-1}} \mathscr{L} Q(s) d \mu(s) h+O\left(h^{2}\right) .
$$

But, by the definition of $\mu$,

$$
\int_{S^{d-1}} \mathscr{L} Q(s) d \mu(s)=0
$$

This ends the proof.

5. Application to an engineering stability problem. Our objective is to study the stability of the motion of a helicopter blade in terms of various physical parameters, such as the velocity of the helicopter, some geometric characteristics of the blade, the intensity or the coloration of the process modeling the turbulence around the blade (this problem was given to us by the French Company Aérospatiale).

5.1. Introduction. In the first approximation, the stability of the movement of a rotor blade with two degrees of freedom is equivalent to the stability of the solution of a linearized ordinary differential equation in $\mathbb{R}^{4}$

$$
\frac{d X(t)}{d t}=A(t) X(t)+F(t)
$$

where the matrix-valued function $A(t)$ and the vector-valued function $F(t)$ are periodic of the same period (the period of rotation of the blade).

When the turbulent flow around the blade is taken into account, we may consider the following linearized model:

$$
\frac{d X(t)}{d t}=A(t) X(t)+F(t)+[B(t) X(t)+G(t)] \sigma(t) \xi^{\varepsilon}(t)
$$

where $B(t)$ (respectively, $G(t)$ ) has the same property as $A(t)$ (respectively, $F(t)$ ), and $\left(\xi^{\varepsilon}(t)\right)$ is a one-dimensional noise. The intensity of the noise $\sigma(t)$ is also a periodic function of the azimuth angle $\Omega t$, where $\Omega$ is the angular velocity of the blade.

All the coefficients $A(t), B(t), F(t), G(t)$ are explicitly known in terms of different physical parameters of the blade.

Here the "stability" we are interested in is the following: the system is stable when it admits a unique periodic in law solution $\{Y(t)\}$ and when, for each initial deterministic condition, the corresponding process $\{X(t)\}$ satisfies

$$
\lim _{t \rightarrow+\infty}|Y(t)-X(t)|=0 \quad \text { a.s. }
$$

First we will consider the white-noise case.

The system (18) beomes

$$
d X(t)=[A(t) X(t)+F(t)] d t+[B(t) X(t)+G(t)] \sigma(t) \circ d W(t)
$$

where $(W(t))$ is a standard one-dimensional Wiener process.

Let us introduce the system

$$
d X(t)=A(t) X(t) d t+B(t) X(t) \sigma(t) \circ d W(t) .
$$

The following theorem can be shown (cf. Pignol [23]). 
THEOREM 5.1. Let us suppose there exists $\lambda<0$ such that the solution of (21) with any deterministic initial condition satisfies

$$
\limsup _{t \rightarrow+\infty} \frac{1}{t} \log |X(t)| \leqq \lambda \quad \text { a.s. }
$$

Then system (20) is stable in the sense of (19).

Of course, it remains to give conditions ensuring the existence of the Lyapunov exponent (22) and to check that the blade system satisfies these conditions. For these points (and the wide-band noise case) we refer to Pignol's thesis [23].

Now let us discuss the numerical computation of the Lyapunov exponent.

We have worked on a set of systems corresponding to different values of some parameters, namely, the distance between the gravity center and the torsion center, the velocity of the helicopter, and the rotation angular velocity.

The numerical values given below correspond to one particular case, but the conclusions are valid for all the situations we have considered.

5.2. The deterministic case. In the deterministic case (corresponding to $\sigma(t) \equiv 0)$, only the Runge-Kutta methods of order larger than 4 give good results (because of the numerical instability of the system, due to the large coefficients of the matrix $A(t)$ and their very short period).

Let us illustrate this remark.

We have used three algorithms:

1. Runge-Kutta method of order 2;

2. Runge-Kutta method of order 4;

3. Gear's method (implemented in the NAG Library of Fortran subroutines).

The discretization step chosen by NAG is always smaller than 0.0016 ; the Lyapunov exponent (in that case, the Floquet exponent) computed is -12.767 .

(1) With a discretization step $h=0.00015$, we obtain the results in Table 1 .

(2) With a discretization step $h=0.003$, we obtain Table 2 .

(3) With a discretization step $h=0.006$, the Floquet exponents are as in Table 3.

TABLE 1

\begin{tabular}{cc}
\hline Method & Floquet exponent \\
\hline Runge-Kutta of order 2 & -12.717 \\
Runge-Kutta of order 4 & -12.767 \\
\hline
\end{tabular}

TABLE 2

\begin{tabular}{cc}
\hline Method & Floquet exponent \\
\hline Runge-Kutta of order 2 & -05.051 \\
Runge-Kutta of order 4 & -12.767 \\
\hline
\end{tabular}

TABLE 3

\begin{tabular}{cc}
\hline Method & Floquet exponent \\
\hline Runge-Kutta of order 2 & +20.154 \\
Runge-Kutta of order 4 & -12.767 \\
\hline
\end{tabular}


These results show that, in the stochastic case, the discretization step will necessarily be chosen smaller than $10^{-4}$.

We will see another consequence.

5.3. Numerical algorithm for the stochastic case. First, it is important to note that the formula $\bar{\lambda}^{h} \sim 1 / N h \log \left|\bar{X}_{N}^{h}\right|$ is of poor interest in practice, because it leads to numerical instabilities, the process $\left(\left|\bar{X}_{p}^{h}\right|\right)$ decreasing to zero or increasing to infinity exponentially fast. Alternatively, the following may be used, which is also used in the proof of Theorem 6.2:

$$
\bar{\lambda}^{h} \sim \frac{1}{N h} \sum_{p=1}^{N} \log \left|M_{p+1}^{h} \bar{s}_{p}^{h}\right|
$$

(with $N$ large; the choice of $N$ will be discussed later).

Now, let us discuss the choice of a convenient discretization algorithm. We have tested the Mil'shtein method and the second-order method (8) on an example due to Baxendale for which there exists an explicit formula giving the Lyapunov exponent.

More precisely, let us consider a one-dimensional Wiener process $(W(t))$ and the following system:

$$
d X(t)=A X(t) d t+\sigma B X(t) \circ d W(t)
$$

with

$$
\begin{aligned}
A & =\left[\begin{array}{ll}
a & 0 \\
0 & b
\end{array}\right], \\
B & =\left[\begin{array}{rr}
0 & -1 \\
1 & 0
\end{array}\right] .
\end{aligned}
$$

Then, we obtain

$$
\lambda=\frac{1}{2}(a+b)+\frac{1}{2}(a-b) \frac{\int_{0}^{2 \pi} \cos (2 \theta) \exp \left(\left(a-b / 2 \sigma^{2}\right) \cos (2 \theta)\right) d \theta}{\int_{0}^{2 \pi} \exp \left(\left(a-b / 2 \sigma^{2}\right) \cos (2 \theta)\right) d \theta} .
$$

For example, let us choose $a=1, b=-2, \sigma=10$. Then an accurate numerical computation gives $\lambda=-0.489$.

For a final integration time $N h=100$ and $h=10^{-3}$, the second-order scheme (8) gives $\lambda=-0.35$, but for $h=10^{-4}$, it gives $\lambda=-0.50$.

The Mil'shteĭn scheme leads to worse results: for the same integration time, and $h=10^{-3}$, it gives $\lambda=3.16$, for $h=10^{-4}$, it gives $\lambda=-0.1$; it is necessary to choose $h=10^{-5}$ to obtain $\lambda=-0.51$ (in that last case, the computation time is much longer than the time due to the second-order scheme with $h=10^{-4}$, for the same result).

Therefore, for our helicopter problem, we have used a second-order scheme. But the above study of the deterministic case shows the necessity to improve scheme (8), in order that it reduces to the Runge-Kutta scheme of order 4 when the intensity of the noise is nought.

Finally, our algorithm has been the following:

1. An initial condition is chosen on the unit sphere $\mathscr{S}^{3}$;

2. At step $(p+1)$, we proceed in two stages:

- The Runge-Kutta method of order 4 is applied to a single step in order to integrate the system

$$
\begin{aligned}
& y(0)=\bar{X}_{p}^{h}, \\
& \dot{y}(t)=\tilde{A}(p h+t) y(t)
\end{aligned}
$$

(the presence of $\tilde{A}$ is due to the discretization of the system written in the Ito sense). 
- Then we calculate

$$
\begin{aligned}
\bar{X}_{p+1}^{h}=y(h)+\{ & \sigma(p h) B \Delta_{p+1}^{h} W+\frac{1}{2} \sigma(p h)^{2} B^{2}\left(\left(\Delta_{p+1}^{h} W\right)^{2}-h\right) \\
& \left.+\frac{1}{2}\left(\sigma(p h)\left(\tilde{A} B+B \tilde{A}+B^{\prime}\right)+\sigma^{\prime}(p h) B\right) h \Delta_{p+1}^{h} W\right\} \bar{X}_{p}^{h}
\end{aligned}
$$

where we have used the following notation: $\left(\Delta_{p+1}^{h} W\right):=$ sequence of mutually independent Gaussian random variables $\mathcal{N}(0, \sqrt{h}), B^{\prime}$ : derivative of the matrix $B(t)$, and all the matrices are calculated at time $t=p h$.

3. The new value of $\lambda$ is computed from the previous one by

$$
\lambda\left(1-\frac{1}{p+1}\right)+\frac{\log \left(\left|\bar{X}_{p+1}^{h}\right|\right)}{(p+1) h}
$$

4. $\bar{X}_{p+1}^{h}$ is projected on the unit sphere.

Just by applying the methods of the previous section, it is easy to check that this algorithm is of order 2 (under hypotheses analogous to that of Theorem 4.2).

Our system of automatic generation of FORTRAN programs of simulation of solutions of Stochastic Differential Systems (see Leblond and Talay [12] for a presentation) can generate the FORTRAN program corresponding to this algorithm, for each bilinear system entered.

5.4. Numerical results. For the given models of blades, the deterministic system was extremely stable for admissible velocities, as shown in Fig. 1, corresponding to one particlar model but typical anyway.

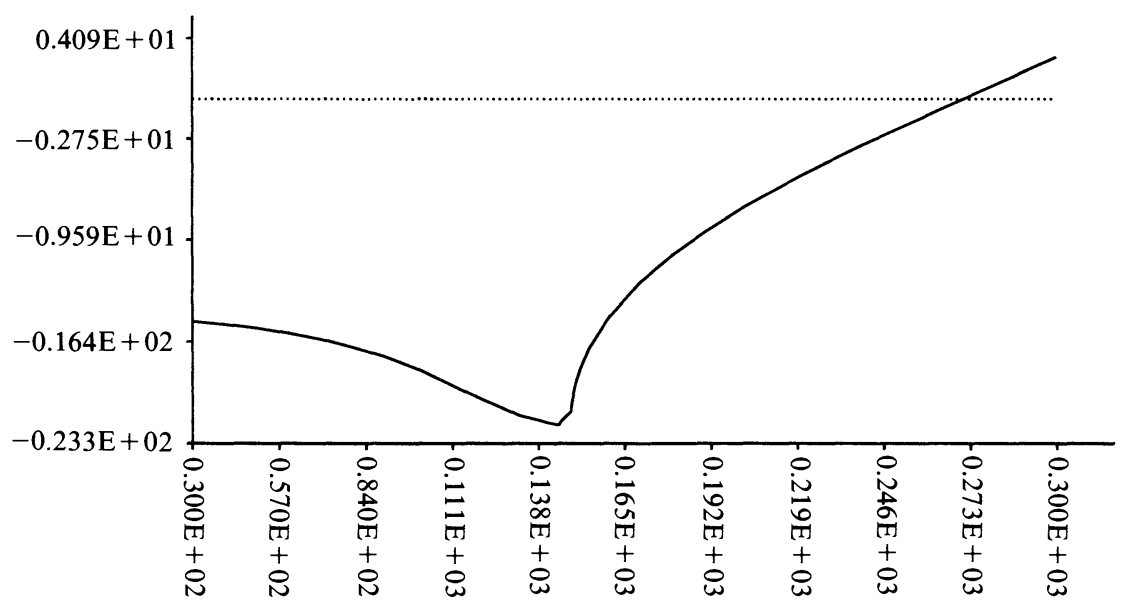

FIG. 1. Variations of the Floquet exponeni in terms of the velocity.

Let us suppose that $\sigma(t)$ is a constant function: $\sigma(t) \equiv \sigma_{0}$ (i.e., the effects of the turbulence are independent of the azimuth angle).

For a velocity equal to $100 \mathrm{~m} / \mathrm{s}$, we obtain the corresponding diagram (see Fig. 2).

The destabilization of the system could occur only for intensities of the noise larger than 0.3; such intensities are not realistic for the turbulent winds around the blade.

Moreover, let us consider a more precise modelization of the noise. We suppose that its intensity is a periodic function of the azimuth angle $\Psi=\Omega t$, reaching its maximum for $\Psi=3 \pi / 4$, and defined by the following function:

$$
\sigma_{0} \exp \left(\delta\left(\cos (0.75 \pi-0.5 \Omega t)^{2}-1\right)\right) \text {. }
$$

Figure 3 represents this function for $\sigma_{0}=0.2$ and $\delta=0.25, \delta=1.00, \delta=2.00$. 


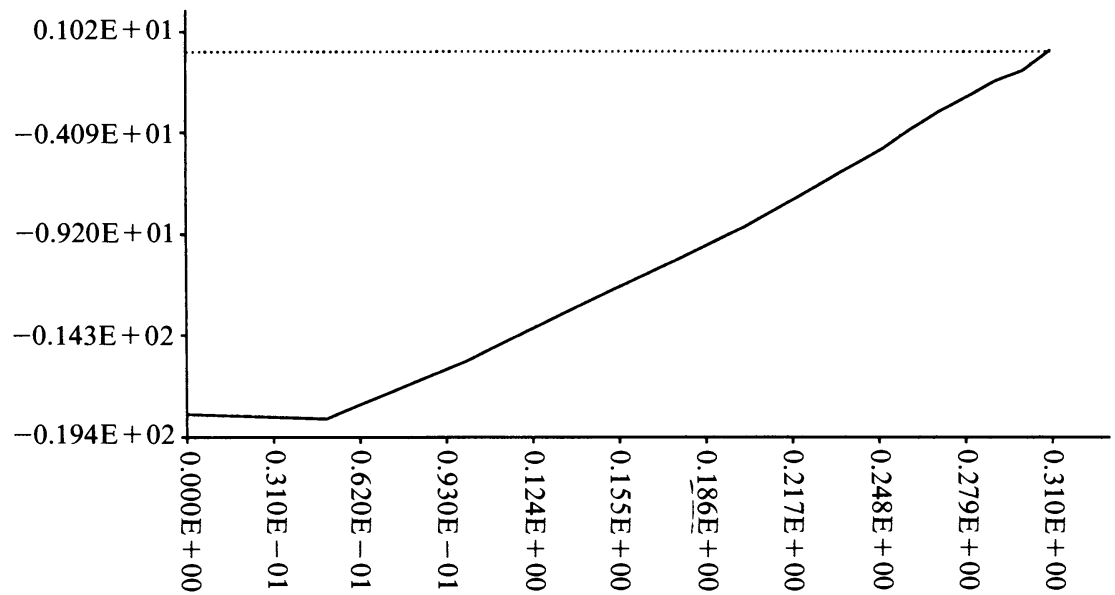

FIG. 2. Variations of the Lyapunov exponent in terms of $\sigma_{0}$.

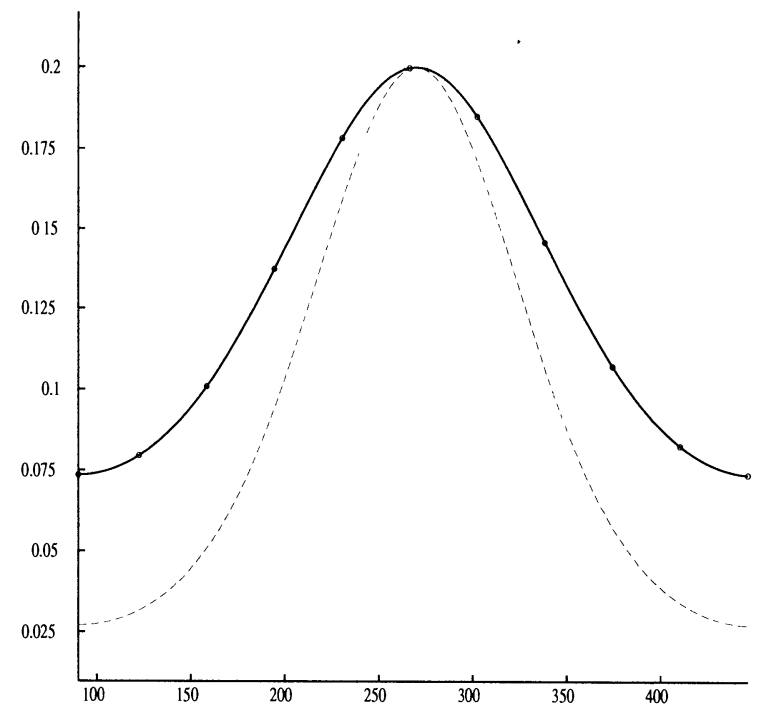

FIG. 3. Variations of the intensity of the noise in terms of the azimuth angle (in degrees).

TABLE 4

\begin{tabular}{clc}
\hline$\sigma_{0}$ & $\delta$ & Lyapunov exponent \\
\hline 0.2 & 0.0 & -8.83 \\
0.2 & 0.25 & -11.83 \\
0.2 & 1.0 & -16.63 \\
0.2 & 2.0 & -18.06
\end{tabular}


In that case, we observe a strong dependency of the Lyapunov exponent upon $\delta$, and the instability could not appear for realistic intensities (see Tables 4 and 5).

5.5. Algorithm for the wideband noise case. Let us consider the system

$$
\frac{d X(t)}{d t}=A(t) X(t)+B(t) X(t) \sigma(t) \xi^{\varepsilon}(t)
$$

with a wideband noise of the form

$$
\xi^{\varepsilon}(t)=\frac{1}{\sqrt{\varepsilon}} Z\left(\frac{t}{\varepsilon}\right)
$$

where $(Z(t))$ is a stationary $\mathcal{N}(0,1)$ Ornstein-Uhlenbeck process.

The above system is an ordinary differential system, so that the pathwise simulation of the solution can be achieved using, for example, the Euler scheme:

$$
\bar{X}_{p+1}^{h}=\bar{X}_{p}^{h}+\left(A(p h) \bar{X}_{p}^{h}+\sigma(p h) B(p h) \xi^{\varepsilon}(p h)\right) \bar{X}_{p}^{h} h .
$$

It is well known that, when $\varepsilon \rightarrow 0$, the solution of (26) converges in law to the solution of (21). Pardoux [18] has shown that, moreover, the Lyapunov exponent of (26) converges to the Lyapunov exponent of (21). Kushner [11] has shown that this convergence is a very particular case of a "pathwise uniform" convergence of a sample average cost per unit time.

But it appears that, even for small $h$, the Lyapunov exponent of that discrete-time process defined by the Euler scheme does not converge, when $\varepsilon \rightarrow 0$, towards the Lyapunov exponent of the system (21).

One reason is that the process $\left\{\xi^{\varepsilon}(p h)\right\}$ does not converge in law, so that the scheme must rather involve the sequence

$$
\Delta_{p+1}^{h} \xi=\int_{p h}^{(p+1) h} \xi^{\varepsilon}(s) d s
$$

which converges in law to the sequence $\left(\Delta_{p+1}^{h} W\right)$.

But it is necessary to be careful: the new scheme

$$
\bar{X}_{p+1}^{h}=\bar{X}_{p}^{h}+\left(A(p h) h+\sigma(p h) B(p h) \Delta_{p+1}^{h} \xi\right) \bar{X}_{p}^{h}
$$

does not converge to a discretization scheme of $(21)(A \neq \tilde{A})$.

Finally, we introduce a convenient second-order scheme, similar to (24).

First, the Runge-Kutta method of order 4 is applied to a single step in order to integrate the system

$$
\begin{aligned}
& y(0)=\bar{X}_{p}^{h}, \\
& \dot{y}(t)=A(p h+t) y(t),
\end{aligned}
$$

TABLE 5

\begin{tabular}{ccc}
\hline$\delta$ & $\sigma_{0}$ & Lyapunov exponent \\
\hline 1.0 & 0.2 & -16.63 \\
1.0 & 0.5 & -6.1 \\
1.0 & 0.6 & -2.11 \\
1.0 & 0.7 & 3.1 \\
\hline
\end{tabular}


and then

$$
\begin{aligned}
\bar{X}_{p+1}^{h}=y(h)+\{ & \sigma(p h) B \Delta_{p+1}^{h} \xi+\frac{1}{2} \sigma(p h)^{2} B^{2}\left(\Delta_{p+1}^{h} \xi\right)^{2} \\
& \left.+\frac{1}{2}\left(\sigma(p h)\left(A B+B A+B^{\prime}\right)+\sigma^{\prime}(p h) B\right) h \Delta_{p+1}^{h} \xi\right\} \bar{X}_{p}^{h} .
\end{aligned}
$$

It is interesting to note that the limit of the above scheme when $\varepsilon \rightarrow 0$ is not the scheme (24), the difference including only terms of order $h \Delta_{p+1}^{h} W$ and $h^{2}$. We have not succeeded in building a second-order scheme of the wideband system converging to a second-order scheme of the white noise system.

Let us describe our simulation of the integrals $\Delta_{p+1}^{h} \xi$.

Let $(V(t))$ be a Wiener process independent of $(W(t))$ such that

$$
d Z(t)=-Z(t) d t+\sqrt{2} d V(t) .
$$

Then we have the formula

$$
\int_{p h}^{(p+1) h} \xi^{\varepsilon}(s) d s=\sqrt{\varepsilon}\left(\frac{1}{2}\left(1-e^{-2 h / \varepsilon}\right) \xi^{\varepsilon}(p h)+\Delta_{p+1}^{1 / \varepsilon}(V)-e^{-2(p+1) h / \varepsilon} \int_{p h}^{(p+1) h} e^{2 s} d V_{s}\right)
$$

Therefore, it is possible to simulate the following vector:

$$
\left(\xi^{\varepsilon}(p h), \Delta_{p+1}^{h} \xi\right)
$$

by the simulation of the Gaussian vector

$$
\left(\Delta_{p+1}^{1 / \varepsilon}(V), \int_{p h}^{(p+1) h} e^{s} d V_{s}, \int_{p h}^{(p+1) h} \cdot e^{2 s} d V_{s}\right)
$$

For our models, we observed that the coloration of the noise tended to stabilize the system (the limit case being the case of very large $\varepsilon$, equivalent to the deterministic case).

5.6. Remarks. The models for the blade and the noise were simplified; in particular, only physical experiments during a flight could permit to improve the modelization of the noise, and overall a more realistic model should be nonlinear.

In this simplified context, the conclusion was that the turbulence around the blade has small effects on the stability of the blade.

5.7. Choice of the integration time. For the sake of simplicity, we again consider the case of matrices $A$ and $B_{j}$ independent of $t$.

As already mentioned, we approximate $\bar{\lambda}^{h}$ by

$$
\bar{\lambda}^{h} \sim \frac{1}{N h} \sum_{p=1}^{N} \log \left|M_{p+1}^{h} \bar{s}_{p}^{h}\right| .
$$

The following Central-limit theorem gives an estimate of the distance between $\lambda$ and (cf. Theorem 2.2)

$$
\frac{1}{t} \int_{0}^{t} Q(s(\theta)) d \theta
$$

(this theorem is due to Bhattacharya [4]).

THEOREM 5.2. Let us suppose $(\mathrm{H})$. Let $\mathscr{L}$ define the infinitesimal generator of the Markov process $(s(t)), \mu$ its unique invariant probability measure, and let the function $Q$ be defined by (4).

For $t \rightarrow+\infty$,

$$
\frac{1}{\sqrt{t}} \int_{0}^{t}(Q(s(\theta))-\lambda) d \theta \rightarrow \mathcal{N}\left(0, \nu^{2}\right) \quad \text { in distribution }
$$




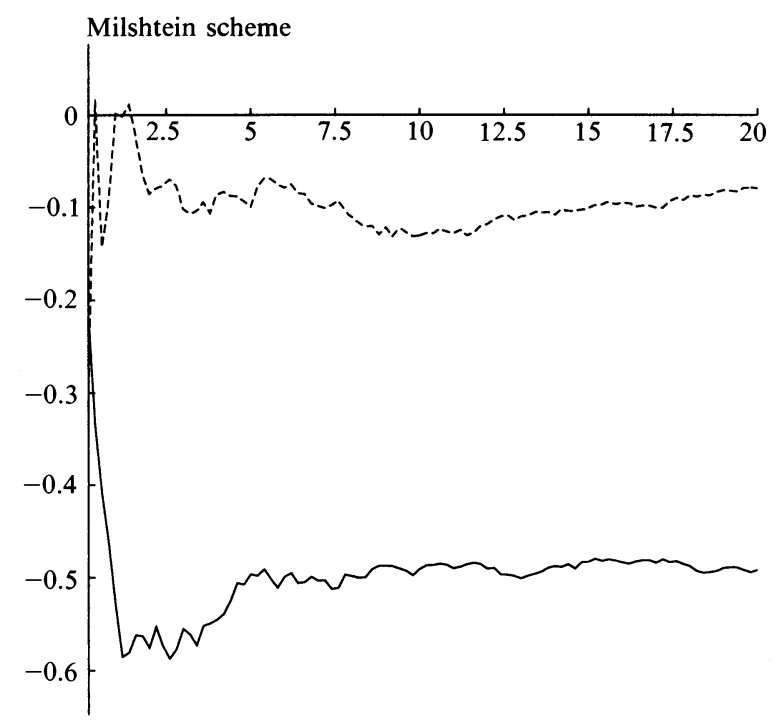

FIG. 4. Variations of the computed value of the Lyapunov exponent.

where the constant $\nu^{2}$ depends only on the coefficients of the system and is given by $(\langle\cdot, \cdot\rangle$ denoting the inner product in $\left.\mathscr{L}^{2}\left(P^{d-1}, \mu\right)\right)$

$$
\nu^{2}=-2\left\langle Q-\lambda, L^{-1}(Q-\lambda)\right\rangle \text {. }
$$

But it seems extremely difficult to numerically estimate the variance of the limit law, since, first, it is necessary to solve a Poisson P.D.E. on $S^{d-1}$, namely,

$$
L u=Q-\lambda,
$$

and, second, since this P.D.E. depends on the unknown $\lambda$.

This important question must be considered in the future. We do not now know a good method to stop the algorithm; in practice, the criterion is to observe that

$$
\frac{1}{N h} \sum_{p=1}^{N} \log \left|M_{p+1}^{h} \bar{s}_{p}^{h}\right|
$$

has become almost constant with respect to $N$ and $h$ after a large number of iterations.

Let us illustrate this point by a figure showing the behaviour of

$$
\frac{1}{N h} \sum_{p=1}^{N} \log \left|M_{p+1}^{h} \bar{s}_{p}^{h}\right|
$$

in terms of $N h$ for $h=10^{-5}$ (continuous line) and $h=10^{-4}$, for the Baxendale's example of $\S 5.3$, with $a=1, b=-2$, and $\sigma=10$ (see Fig. 4). We recall that the true value of $\lambda$ is -0.489 .

6. Appendix: second-order schemes.

6.1. Preliminaries. Let us consider the general stochastic differential equation in the Ito sense:

$$
d X(t)=b(X(t)) d t+\sigma(X(t)) d W(t) .
$$

The Euler scheme is defined by

$$
\bar{X}_{p+1}^{h}=\bar{X}_{p}^{h}+\sum_{j=1}^{r} \sigma_{j}\left(\bar{X}_{p}^{h}\right) U_{p+1}^{j} \sqrt{h}+b\left(\bar{X}_{p}^{h}\right) h .
$$


The Mil'shteĭn scheme is defined by

$$
\bar{X}_{p+1}^{h}=\bar{X}_{p}^{h}+\sum_{j=1}^{r} \sigma_{j}\left(\bar{X}_{p}^{h}\right) U_{p+1}^{j} \sqrt{h}+b\left(\bar{X}_{p}^{h}\right) h+\sum_{j, k=1}^{r} \partial \sigma_{j}\left(\bar{X}_{p}^{h}\right) \sigma_{k}\left(\bar{X}_{p}^{h}\right) S_{p+1}^{k j} h .
$$

We define the matrix $a$ and the vectors $A_{j}$ by

$$
\begin{aligned}
& a=\sigma \sigma^{*}, \\
& A_{j}=\frac{1}{2} \sum_{k, l=1}^{d} a_{l}^{k} \partial_{k l} \sigma_{j} .
\end{aligned}
$$

Besides, we will denote by $L$ the infinitesimal generator of the process $(X(t))$ :

$$
L=\sum_{i=1}^{d} b^{i} \partial_{i}+\frac{1}{2} \sum_{i=1}^{d} \sum_{j=1}^{d} a_{j}^{i} \partial_{i j}
$$

Now, we define a new scheme by

$$
\begin{aligned}
\bar{X}_{p+1}^{h}=\bar{X}_{p}^{h}+ & \sum_{j=1}^{r} \sigma_{j}\left(\bar{X}_{p}^{h}\right) U_{p+1}^{j} \sqrt{h}+b\left(\bar{X}_{p}^{h}\right) h+\sum_{j, k=1}^{r} \partial \sigma_{j}\left(\bar{X}_{p}^{h}\right) \sigma_{k}\left(\bar{X}_{p}^{h}\right) S_{p+1}^{k j} h \\
& +\frac{1}{2} \sum_{j=1}^{r}\left\{\partial b\left(\bar{X}_{p}^{h}\right) \sigma_{j}\left(\bar{X}_{p}^{h}\right)+\partial \sigma_{j}\left(\bar{X}_{p}^{h}\right) b\left(\bar{X}_{p}^{h}\right)+A_{j}\left(\bar{X}_{p}^{h}\right)\right\} U_{p+1}^{j} h^{3 / 2} \\
& +\frac{1}{2} L b\left(\bar{X}_{p}^{h}\right) h^{2} .
\end{aligned}
$$

Scheme (8) is a particular application of this scheme.

6.2. Second-order discretization schemes. In this section, $\mathscr{F}_{p}$ will be the $\sigma$-algebra generated by $\left(\bar{X}_{0}^{h}, \cdots, \bar{X}_{p}^{h}\right)$.

A discretization scheme will be called of second order if it satisfies (all the right-side terms of the equalities being understood evaluated at $\bar{X}_{p}^{h}$, and using the usual convention for the summation indices)

$$
\begin{aligned}
& E\left(\Delta_{p+1}^{h} \bar{X} \mid \mathscr{F}_{p}\right)=b h+\frac{1}{2}(L b) h^{2}+\xi_{p+1}, \quad E\left|\xi_{p+1}\right| \leqq C h^{3}, \\
& \left.E\left(\left(\Delta_{p+1}^{h} \bar{X}\right)^{i_{1}}\left(\Delta_{p+1}^{h} \bar{X}\right)^{i_{2}}\right) \mid \mathscr{F}_{p}\right) \\
& =\sigma_{j}^{i_{1}} \sigma_{j}^{i_{2}} h+\left(b^{i_{1}} b^{i_{2}}+\frac{1}{2} \partial_{k_{1}} \sigma_{j}^{i_{1}} \partial_{k_{2}} \sigma_{j}^{i_{2}} \sigma_{l}^{k_{1}} \sigma_{l}^{k_{2}}+\frac{1}{2} \partial_{k} b^{i_{2}} \sigma_{j}^{i_{1}} \sigma_{j}^{k}+\frac{1}{2} \partial_{k} b^{i_{1}} \sigma_{j}^{i_{2}} \sigma_{j}^{k}\right. \\
& \left.+\frac{1}{2} \sigma_{j}^{i_{1} \partial_{k}} \sigma_{j}^{i_{2}} b^{k}+\frac{1}{2} \sigma_{j}^{i_{2}} \partial_{k} \sigma_{j}^{i_{1}} b^{k}+\frac{1}{4} \sigma_{j}^{i_{1}} \partial_{k l} \sigma_{j}^{i_{2}} \sigma_{n}^{k} \sigma_{n}^{l}+\frac{1}{4} \sigma_{j}^{i_{2}} \partial_{k l} \sigma_{j}^{i_{1}} \sigma_{n}^{k} \sigma_{n}^{l}\right) h^{2} \\
& +\xi_{p+1}^{i_{1} i_{2}}, \quad E\left|\xi_{p+1}^{i_{1} i_{2}}\right| \leqq C h^{3}, \\
& \left.E\left(\left(\Delta_{p+1}^{h} \bar{X}\right)^{i_{1}} \ldots\left(\Delta_{p+1}^{h} \bar{X}\right)^{i_{3}}\right) \mid \mathscr{F}_{p}\right) \\
& =\left(b^{i_{1}} \sigma_{j}^{i_{2}} \sigma_{j}^{i_{3}}+b^{i_{2}} \sigma_{j}^{i_{3}} \sigma_{j}^{i_{1}}+b^{i_{3}} \sigma_{j}^{i_{1}} \sigma_{j}^{i_{2}}+\frac{1}{2} \sigma_{l}^{i_{2}} \partial_{k} \sigma_{l}^{i_{3}} \sigma_{j}^{i_{1}} \sigma_{j}^{k}+\frac{1}{2} \sigma_{l}^{i_{3}} \partial_{k} \sigma_{l}^{i_{2}} \sigma_{j}^{i_{1}} \sigma_{j}^{k}\right. \\
& \left.+\frac{1}{2} \sigma_{l}^{i_{3}} \partial_{k} \sigma_{l}^{i_{1}} \sigma_{j}^{i_{2}} \sigma_{j}^{k}+\frac{1}{2} \sigma_{l}^{i_{1}} \partial_{k} \sigma_{l}^{i_{3}} \sigma_{j}^{i_{2}} \sigma_{j}^{k}+\frac{1}{2} \sigma_{l}^{i_{1}} \partial_{k} \sigma_{l}^{i_{2}} \sigma_{j}^{i_{3}} \sigma_{j}^{k}+\frac{1}{2} \sigma_{l}^{i_{2}} \partial_{k} \sigma_{j}^{i_{1}} \sigma_{j}^{i_{3}} \sigma_{j}^{k}\right) h^{2} \\
& +\xi_{p+1}^{i_{1} i_{2} i_{3}}, \quad E\left|\xi_{p+1}^{i_{1} i_{2} i_{3}}\right| \leqq C h^{3}, \\
& \left.E\left(\left(\Delta_{p+1}^{h} \bar{X}\right)^{i_{1}} \cdots\left(\Delta_{p+1}^{h} \bar{X}\right)^{i_{4}}\right) \mid \mathscr{F}_{p}\right) \\
& =\left(\sigma_{j}^{i_{1}} \sigma_{j}^{i_{2}} \sigma_{l}^{i_{3}} \sigma_{l}^{i_{4}}+\sigma_{j}^{i_{1}} \sigma_{j}^{i_{4}} \sigma_{l}^{i_{2}} \sigma_{l}^{i_{3}}\right) h^{2}+\xi_{p+1}^{i_{1} \cdots i_{4}}, \quad E\left|\xi_{p+1}^{i_{1} \cdots i_{4}}\right| \leqq C h^{3}, \\
& \left.E\left(\left(\Delta_{p+1}^{h} \bar{X}\right)^{i_{1}} \cdots\left(\Delta_{p+1}^{h} \bar{X}\right)^{i_{5}}\right) \mid \mathscr{F}_{p}\right)=\xi_{p+1}^{i_{1} \cdots i_{5}}, \quad E\left|\xi_{p+1}^{i_{1} \cdots i_{5}}\right| \leqq C h^{3}, \\
& \left.E\left(\left(\Delta_{p+1}^{h} \bar{X}\right)^{i_{1}} \cdots\left(\Delta_{p+1}^{h} \bar{X}\right)^{i_{6}}\right) \mid \mathscr{F}_{p}\right)=\xi_{p+1}^{i_{1} \cdots i_{6}}, \quad E\left|\xi_{p+1}^{i_{1} \cdots i_{6}}\right| \leqq C h^{3} .
\end{aligned}
$$

We have the following theorem (see Talay [27] or [28], Mil'shteĭn [16]).

THEOREM 6.1. Let us suppose that $b$ and $\sigma$ are smooth functions with all their derivatives bounded, and only (i) of (HU). 
Then, for all functions $f$ of class $\mathscr{C}^{\infty}$ such that $f$ and all its derivatives have an at most polynomial growth at infinity, the Euler and Mil'shtern schemes with $\bar{X}_{0}^{h}=X(0)$ satisfy, for any $h$ small enough, for any $T>0$, and any $h=T / N\left(N \in \mathbb{N}^{*}\right)$,

$$
\forall p=1, \cdots, N:\left|E f\left(X_{p h}\right)-E f\left(\bar{X}_{p}^{h}\right)\right| \leqq C h,
$$

whereas scheme (30) satisfies, as well as any other second-order scheme such that $\bar{X}_{p}^{h}$ has all its moments finite for all $p$ (under the condition $\bar{X}_{0}^{h}=X(0)$ ),

$$
\left|E f\left(X_{p h}\right)-E f\left(\bar{X}_{p}^{h}\right)\right| \leqq C h^{2} .
$$

The same respective orders of convergence are conserved (under another set of hypotheses) when the process $(X(t))$ has a unique invariant measure $\mu$ and the criterion of convergence is

$$
\mid \int f(\theta) d \mu(\theta)-\text { a.s. } \lim _{N \rightarrow+\infty} \frac{1}{N} \sum_{p=1}^{N} f\left(\bar{X}_{p}^{h}(x)\right) \mid
$$

(see Talay [29]).

6.3. Extension of the main theorem. Following the same procedure as previously, we may extend the results of Theorem 4.2 to second-order schemes, under some conditions concerning the law of the involved random variables.

THEOREM 6.2. Let us suppose that system (3) satisfies Hypothesis (H0).

Let $\left(\bar{X}_{p}^{h}, p \in \mathbb{N}\right)$ be a discretization scheme of the form

$$
\bar{X}_{p+1}^{h}=M_{p+1}^{h} \bar{X}_{p}^{h} \text {. }
$$

We suppose that there exists $h_{0}>0$ such that the following hypotheses hold.

(H1) For any $h<h_{0},\left(M_{p}^{h}\right)$ is a sequence of independently and identically distributed random matrices in $G l\left(\mathbb{R}^{d}\right)$, with common distribution $\nu^{h}$.

(H2) For any $h<h_{0}$,

$$
E \log ^{+}\left|M_{1}^{h}\right|<+\infty, \quad E \log ^{+}\left|\left(M_{1}^{h}\right)^{-1}\right|<+\infty .
$$

(H3) For any $h<h_{0}$, the smallest subgroup of $G l\left(\mathbb{R}^{d}\right)$ containing the support of $\nu^{h}$ is irreducible, and, moreover, the process $\left(\bar{X}_{p}^{h} /\left|\bar{X}_{p}^{h}\right|\right)$ is ergodic on $S^{d-1}$.

(H4) There exists $C(h)$ such that, almost surely, the distance between $x$ and $M_{1}^{h} x$ can be bounded by $C(h)$ uniformly in $x$ in $S^{d-1}$, and $C(h) \rightarrow 0$ with $h$.

Let us suppose: $\left(\bar{X}_{p}^{h}, p \in \mathbb{N}\right)$ is a second-order scheme in the sense of $\S 6.2$.

Then (i) There exists a real number $\bar{\lambda}^{h}$ such that

$$
\forall x \in \mathbb{R}^{d}-\{0\}: \bar{\lambda}^{h}=\lim _{p \rightarrow+\infty} \frac{1}{p h} \log \left|\bar{X}_{p}^{h}(x)\right|, \quad \text { a.s. }
$$

(ii) Moreover, if $\lambda$ is the upper Lyapunov exponent of (1),

$$
\left|\lambda-\bar{\lambda}^{h}\right|=O\left(h^{2}\right) .
$$

7. Conclusion. We have built schemes which are of second-order for the approximation of the upper Lyapunov exponents of bilinear systems.

These schemes have been tested numerically and have served to solve an industrial problem of stability.

Some aspects are still to be improved, for example, the choice of the computation time.

Finally, let us point out that the approximation of the Lyapunov spectrum of nonlinear systems on compact manifolds is studied in Grorud and Talay [9], and that an extrapolation technique based upon the Euler scheme permits us to obtain a second-order accuracy (Talay and Tubaro [30]). 
Acknowledgment. The author thanks Luciano Tubaro of the University of Trento for very helpful discussions and comments.

\section{REFERENCES}

[1] L. Arnold, W. Kliemann, And E. Oeljeklaus, Lyapunov exponent of a linear stochastic system, in Lyapunov Exponents, L. Arnold and V. Wihstutz, eds., Lecture Notes in Math. 1186, SpringerVerlag, Berlin, New York, 1986.

[2] L. Arnold, E. Oeljeklaus, And E. Pardoux, Amost sure and moment stability for linear Ito equations, in Lyapunov Exponents, L. Arnold and V. Wihstutz, eds., Lecture Notes in Math. 1186, Springer-Verlag, Berlin, New York, 1986.

[3] E. I. Auslender And G. N. Mil'ShteǏn, Asymptotic expansion of the Lyapunov index for linear stochastic systems with small noise, Prikl. Mat. Mekh., 46 (1982), pp. 358-365.

[4] R. N. BhAtTACHARyA, On the functional central limit theorem and the law of the iterated logarithm for Markov processes, Z. Wahrscheinlichkeitstheorie verw. Gebiete, 60 (1982), pp. 185-201.

[5] P. BOUGEROL AND J. LACROIX, Products of random matrices with applications to Schrödinger operators, Birkhäuser, Boston, 1985.

[6] J. M. C. CLARK AND R. J. CAMERON, The maximum rate of convergence of discrete approximations for stochastic differential equations, Stochastic Differential Systems-Filtering and Control, B. Grigelionis, ed., Proceedings of the IFIP Working Conference, Vilnius, Lithuania, USSR, 1978, Lecture Notes in Control Inform. Sci. 25, Springer-Verlag, Berlin, New York, 1980.

[7] J. M. C. Clark, An efficient approximation for a class of stochastic differential equations, in Advances in Filtering and Optimal Stochastic Control, W. Fleming and L. Gorostiza, eds., Proceedings of the IFIP Working Conference, Cocoyoc, Mexico, 1982, Lecture Notes in Control Inform. Sci. 42, Springer-Verlag, Berlin, New York, 1982.

[8] J. L. Doob, Stochastic Processes, John Wiley, New York, 1953.

[9] A. Grorud AND D. TALAY, Approximation of Lyapunov exponents of stochastic differential systems on compact manifolds, 9th Conference on Analysis and Optimization of Systems, A. Bensoussan and J. L. Lions, eds., Lecture Notes in Control Inform. Sci. 144, Springer-Verlag, Berlin, New York, 1990.

[10] N. Ikeda and S. Watanabe, Stochastic Differential Equations and Diffusion Processes, NorthHolland, Amsterdam, 1981.

[11] H. J. KuSHNER, Approximations and optimal control for the pathwise average cost per unit time and discounted problems for wideband noise driven systems, SIAM J. Control Optimi., 27 (1987), pp. 546-562.

[12] J. Leblond And D. TAlay, Simulation of diffusion processes with Presto, building systems like Presto with Adagio, Proceedings of the 7th French-Belgian Statisticians Meeting, Rouen, France, 1986.

[13] F. Ledrappier, Quelques propriétés des exposants caractréristiques, Cours de l'Ecole d'été de Probabilités de Saint-Flour 1982, Lecture Notes in Math., Springer-Verlag, Berlin, New York, 1984.

[14] G. N. Mı́'shteĭn, Approximate integration of stochastic differential equations, Theory of Probability and Applications, 19 (1974), pp. 557-562.

[15] — A method of second-order accuracy integration of stochastic differential equations, Theory Probab. Appl., 23 (1976), pp. 414-419.

[16] - Weak approximation of solutions of systems of stochastic differential equations, Theory Probab. Appl., 30 (1985), pp. 750-766.

[17] N. J. NEwTON, An asymptotically efficient difference formula for solving stochastic differential equations, Stochastics, 19 (1986), pp. 175-206.

[18] E. Pardoux, Wide-band limit of Lyapunov exponents, Proc. of the 3rd Bad Honnef Conference on Stochastic Differential Systems, N. Christopeit, K. Helmes, and M. Kohlmann, eds., Lecture Notes in Control Inform. Sci. 78, Springer-Verlag, Berlin, New York, 1986.

[19] E. PARDOUX AND M. PIGNOL, Etude de la stabilité de la solution d'une E.D.S. bilinéaire à coefficients périodiques. Application au mouvement d'une pale d'hélicoptère, in Analysis and Optimization of Systems, Part 2, A. Bensoussan and J. L. Lions, eds., Lecture Notes in Control Inform. Sci., 63 (1984), pp. 92-103.

[20] E. Pardoux And D. TAlay, Discretization and simulation of S.D.E., Acta Appl. Math., 3 (1985), p. 23-47.

[21] - Stability of linear differential systems with parametric excitation, Nonlinear Stochastic Dynamic Engineering Systems, G. I. Schueller and F. Ziegler, eds., Proceedings of the IUTAM Symposium, Innsbruck, 1987, Springer-Verlag, Berlin, New York, 1988. 
[22] E. PARdoux AND V. Wihstutz, Lyapunov exponent of linear stochastic systems with large diffusion term, SIAM J. Appl. Math., 48 (1988), pp. 442-457.

[23] M. Pignol, Stabilité stochastique des pales d'hélicoptère, Thèse de 3ème Cycle, Université de Provence, France, 1985.

[24] E. Platen, An approximation method for a class of Ito processes, Lietuvos Mat. Rinkiniys, XXI (1981), pp. 121-133.

[25] W. RuMELIN, Numerical treatment of stochastic differential equations, SIAM J. Numer. Anal., 19 (1982), pp. 604-613.

[26] D. TALAY, Résolution trajectorielle et analyse numérique des équations différentielles stochastiques, Stochastics, 9 (1983), pp. 275-306.

[27] — Efficient numerical schemes for the approximation of expectations of functionals of S.D.E., in Filtering and Control of Random Processes, H. Korezlioglu, G. Mazziotto, and J. Szpirglas, eds., Proceedings of the ENST-CNET Colloquium, Paris, 1983, Lecture Notes in Control Inform. Sci., 61, Springer-Verlag, Berlin, New York, 1984.

[28] — Discretisation d'une E.D.S. et calcul approaché d'espérances de fonctionnelles de la solution, RAIRO Modél. Math. Anal. Numér., 20 (1986), p. 141.

[29] - Second-order discretization schemes of stochastic differential systems for the computation of the invariant law, Stochastics Stochastics Rep., 29 (1990), pp. 13-36.

[30] D. TALAY AND L. TUBARO, Expansion of the global error for numerical schemes solving stochastic differential equations, Stochastic Anal. Appl., 8 (1990), pp. 94-120. 